\title{
Electric field tuning of the band gap in graphene multilayers
}

\author{
A. A. Avetisyan, ${ }^{*}$ B. Partoens ${ }^{\dagger}$ and F. M. Peeters ${ }^{\ddagger}$ \\ Departement Fysica, Universiteit Antwerpen, Groenenborgerlaan 171, B-2020 Antwerpen, Belgium \\ (Received 18 September 2008; revised manuscript received 8 December 2008; published 29 January 2009)

\begin{abstract}
A perpendicular electric field applied to multilayers of graphene modifies the electronic structure near the $K$ point and may induce an energy gap in the electronic spectrum. This gap is tunable by the gate voltage and its size depends on the number of layers. We use a tight-binding approach to calculate the band structure and include a self-consistent calculation in order to obtain the density of charge carriers. Results are presented for systems consisting of three and four layers of graphene. The effect of the circular asymmetry of the band structure on the gap is critically examined.
\end{abstract}

DOI: 10.1103/PhysRevB.79.035421

PACS number(s): 81.05.Uw, 73.63.Bd, 73.43.Cd

\section{INTRODUCTION}

Graphene is a two-dimensional (2D) crystal of carbon atoms which was fabricated only very recently. ${ }^{1}$ Since then it has become an important research area in materials science and condensed-matter physics. Already, a huge amount of theoretical and experimental papers have been devoted to the investigation of the low-energy electronic properties of ultrathin graphite films, including graphene monolayers and bilayers. This activity is stimulated by the successful fabrication of stable ultrathin graphite films, which exhibit excellent mechanical quality, exotic energetic spectrum and are very promising for, e.g., nanoelectronics ${ }^{2}$ and as transparent conducting layers ${ }^{3}$ which are important for, e.g., displays and solar cells. Rich electronic structures and optical properties are predicted in such systems.

Based on angle-resolved photoemission spectroscopy (ARPES) experiments ${ }^{4}$ and an analysis of the quantum Hall effect and Shubnikov-de Haas oscillations, it was concluded that in a single graphene layer the carriers have a linear Dirac-like spectrum around the Fermi energy. ${ }^{1,5,6}$ Also in quasi-2D highly oriented pyrolytic graphite (HOPG) both conventional electrons with parabolic dispersion and Diraclike electrons with linear spectrum were observed. ${ }^{7}$

Within a tight-binding approach it was shown ${ }^{8}$ that Dirac fermions are present in $A B$ stacked graphene multilayers with an odd number of layers and that for an even number of stacked graphene layers, only normal fermions with a parabolic energy dispersion are found near the $K$ point. This approach, as well as $a b$ initio density-functional calculations, ${ }^{9}$ was used earlier to investigate the evolution of the electronic structure around the $K$ point when we go from a single graphene layer to bulk graphite. ${ }^{10}$

A serious drawback of the use of a single layer of graphene for electronics is the observation that conduction cannot be switched off. ${ }^{11}$ It has been shown that this can be overcome by using a bilayer of graphene where a perpendicular electric field induces an energy gap at the $K$ point. Theoretical and experimental investigations of bilayer graphene have shown that its band structure can be controlled by an applied electric field directed perpendicular to the layers, so that the electronic gap between the valence and conduction bands can be tuned between zero and midinfrared energies. ${ }^{12,13}$ McCann $^{12}$ showed theoretically that in bilayer graphene the asymmetry between on-site energies in the layers, caused by an external gate, leads to a gap between the otherwise degenerate conduction and valence bands. It is expected that a similar energy gap can be opened in multilayers of graphene and the value of the gap will depend on the number of the layers. Such a theoretical study has not been made yet and the purpose of the present paper is to present an investigation of the electron spectrum of three and four layers of graphene near the $K$ point in the presence of an external electric field. In this paper we will show that by applying a perpendicular electric field an energy gap can be induced in multilayer graphene which is tunable and which depends on the number of layers. In contrast to the bilayer case, we found that the size of the energy gap is not a monotonous function of the gate voltage. We show that for certain values of the gate voltage the gap may even close both for the three- and four-layer graphene systems. Our analysis is based on a tight-binding approach to calculate the band structure of three- and four-layer graphene systems in the case a gate voltage is applied. A self-consistent Hartree approximation is used to calculate the induced charges on the different graphene layers. Such three-layer graphene samples can be realized experimentally, as was recently demonstrated in Ref. 14, in which transport measurements on a tunable three-layer graphene single-electron transistor were reported, and its functionality was proven by Coulomb blockade oscillations.

This paper is organized as follows. In Sec. II the details of our tight-binding approach are given, with the description of the self-consistent calculation performed in Sec. II A for a three-layer graphene system and in Sec. II B for a four-layer graphene system, in the presence of an external electric field. In Sec. III our numerical results are presented and Sec. IV summarizes our conclusions.

\section{THEORETICAL FORMALISM}

Multilayer graphene is modeled as coupled hexagonal lattices with inequivalent sites $A_{i}$ and $B_{i}$ in the $i$ th layer, with $A_{i}$ and $A_{i+1}$ atoms in adjacent layers on top of each other. The tight-binding Hamiltonian for an arbitrary number of $A B$ stacked layers was presented in Ref. 10 and has the form 


$\left(\begin{array}{cc|cc|cc|cc|c}\Delta+\gamma_{5} & \gamma_{0} f & \gamma_{1} & -\gamma_{4} f^{*} & \gamma_{5} / 2 & 0 & 0 & 0 & \cdots \\ \gamma_{0} f^{*} & \gamma_{2} & -\gamma_{4} f^{*} & \gamma_{3} f & 0 & \gamma_{2} / 2 & 0 & 0 & \cdots \\ \hline \gamma_{1} & -\gamma_{4} f & \Delta+\gamma_{5} & \gamma_{0} f^{*} & \gamma_{1} & -\gamma_{4} f & \gamma_{5} / 2 & 0 & \cdots \\ -\gamma_{4} f & \gamma_{3} f^{*} & \gamma_{0} f & \gamma_{2} & -\gamma_{4} f & \gamma_{3} f^{*} & 0 & \gamma_{2} / 2 & \cdots \\ \hline \gamma_{5} / 2 & 0 & \gamma_{1} & -\gamma_{4} f^{*} & \Delta+\gamma_{5} & \gamma_{0} f & \gamma_{1} & -\gamma_{4} f^{*} & \cdots \\ 0 & \gamma_{2} / 2 & -\gamma_{4} f^{*} & \gamma_{3} f & \gamma_{0} f^{*} & \gamma_{2} & -\gamma_{4} f^{*} & \gamma_{3} f & \cdots \\ \hline 0 & 0 & \gamma_{5} / 2 & 0 & \gamma_{1} & -\gamma_{4} f & \Delta+\gamma_{5} & \gamma_{0} f^{*} & \cdots \\ 0 & 0 & 0 & \gamma_{2} / 2 & -\gamma_{4} f & \gamma_{3} f^{*} & \gamma_{0} f & \gamma_{2} & \cdots \\ \hline \vdots & \vdots & \vdots & \vdots & \vdots & \vdots & \vdots & \vdots & \ddots\end{array}\right)$,

where the rows and columns are ordered according to atom $A$ from layer 1, atom $B$ from layer 1, atom $A$ from layer 2 , atom $B$ from layer 2, etc. In Eq. (1) $\gamma_{0}, \gamma_{1}, \gamma_{2}, \gamma_{3}, \gamma_{4}, \gamma_{5}$, and $\Delta$ are the Slonczewski-Weiss-McClure (SWMcC) parameters and the function $f$ stands for

$$
f\left(k_{x}, k_{y}\right)=e^{i k_{x} a_{0} / \sqrt{3}}+2 e^{-i k_{x} a_{0} / 2 \sqrt{3}} \cos k_{y} a_{0} / 2,
$$

with $a_{0}=2.46 \AA$ as the length of the in-plane lattice vector. The six parameters $\gamma_{0}, \gamma_{1}, \gamma_{2}, \gamma_{3}, \gamma_{4}$, and $\gamma_{5}$ express the couplings between the different atoms and are given in Ref. 10.

\section{A. Three-layer graphene system in an external electric field}

First, we consider a system consisting of three layers of graphene positioned on top of a positively charged gate, as shown in Fig. 1, which is employed to control the density of electrons $n$ on the three-layer system. The gate with a positive charge density $n$ induces a total excess density $n=n_{1}$ $+n_{2}+n_{3}$, where $n_{1}$ is the excess density on the closest layer to the gate and $n_{2}\left(n_{3}\right)$ is the excess density on the second (third) layer from the gate. In our model the gate produces a uniform electric field $E=n e / 2 \varepsilon_{0} \kappa$, where $\varepsilon_{0}$ is vacuum permittivity and $\kappa$ the dielectric constant where the value $\kappa=1$ is used in the paper for our numerical calculations. The layers of graphene, in its turn, produce a uniform electric field $E_{i}$ $=n_{i} e / 2 \varepsilon_{0} \kappa$, where $i=1,2,3$ is the layer number. The layer asymmetries between first and second layers, as well as between second and third layers, are determined by a corresponding change in the potential energy $\Delta_{1,2}\left(\Delta_{2,3}\right)$,

$$
\Delta_{1,2}(n)=\alpha\left(n_{2}+n_{3}\right),
$$

and

$$
\Delta_{2,3}(n)=\alpha n_{3},
$$

where $\alpha=e^{2} c_{0} / \varepsilon_{0} \kappa$, with $c_{0}=3.35 \AA$ as the interlayer distance.

The Hamiltonian for the three $A B A$ stacked graphene layers in the presence of an external electric field can be obtained by adding $-\Delta_{1,2}(n)$ and $\Delta_{2,3}(n)$ to the first and third layer on-site elements of Eq. (1), respectively,

$$
H=\left(\begin{array}{cc|cc|cc}
-\Delta_{1,2}(n)+\Delta+\gamma_{5} & \gamma_{0} f & \gamma_{1} & -\gamma_{4} f^{*} & \gamma_{5} / 2 & 0 \\
\gamma_{0} f^{*} & -\Delta_{1,2}(n)+\gamma_{2} & -\gamma_{4} f^{*} & \gamma_{3} f & 0 & \gamma_{2} / 2 \\
\hline \gamma_{1} & -\gamma_{4} f & \Delta+\gamma_{5} & \gamma_{0} f^{*} & \gamma_{1} & -\gamma_{4} f \\
-\gamma_{4} f & \gamma_{3} f^{*} & \gamma_{0} f & \gamma_{2} & -\gamma_{4} f & \gamma_{3} f^{*} \\
\hline \gamma_{5} / 2 & 0 & \gamma_{1} & -\gamma_{4} f^{*} & \Delta_{2,3}(n)+\Delta+\gamma_{5} & \gamma_{0} f \\
0 & \gamma_{2} / 2 & -\gamma_{4} f^{*} & \gamma_{3} f & \gamma_{0} f^{*} & \Delta_{2,3}(n)+\gamma_{2}
\end{array}\right) .
$$

The tight-binding Hamiltonian operates in the space of coefficients of the tight-binding functions $c(\vec{k})$ $=\left(c_{A_{1}}, c_{B_{1}}, c_{A_{2}}, c_{B_{2}}, c_{A_{3}}, c_{B_{3}}\right)$, where $c_{A_{i}}=c_{A_{i}}(\vec{k})$ and $c_{B_{i}}$ $=c_{B_{i}}(\vec{k})$ are the $i$ th layer coefficients for $A$ and $B$ types of atoms, respectively. The total eigenfunction of the system is then given by

$$
\begin{aligned}
& \qquad \Psi_{\vec{k}}(\vec{r})=\sum_{i=1}^{N_{l}} c_{A_{i}} \psi_{\vec{k}}^{A_{i}}(\vec{r})+\sum_{i=1}^{N_{l}} c_{B_{i}} \psi_{\vec{k}}^{B_{i}}(\vec{r}), \\
& \text { with } N_{l} \text { as the number of layers. The six coefficients in Eq. } \\
& \text { (6) of the three-layer system, for fixed values of the layer }
\end{aligned}
$$
asymmetries defined by Eqs. (3) and (4), can be obtained by 

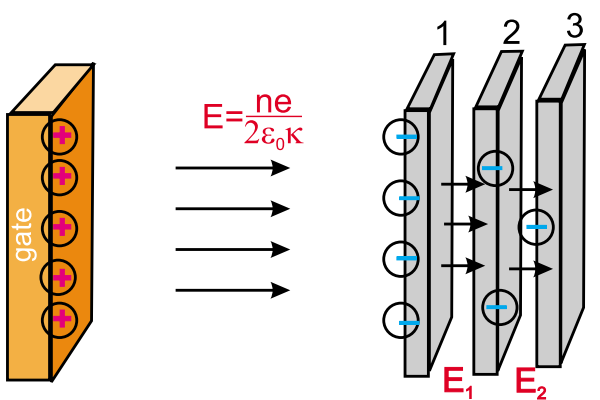

FIG. 1. (Color online) Schematic of the three-layer graphene system near a positively charged gate, which induces a total excess density $n=n_{1}+n_{2}+n_{3}$, with $n_{1}$ the excess density of electrons on the closest layer to the gate, and $n_{2}\left(n_{3}\right)$ is the excess density on the second (third) layer; $E_{i}(i=1,2)$ is the uniform electric field between the layers with $E_{1}=\left(n_{2}+n_{3}\right) e / \varepsilon_{0} \kappa$ and $E_{2}=\left(n_{3}\right) e / \varepsilon_{0} \kappa$.

diagonalizing Eq. (5). The electronic densities on the individual layers are given by

$$
n_{i}=\frac{2}{\pi} \int d k_{x} d k_{y}\left(\left|c_{A_{i}}\right|^{2}+\left|c_{B_{i}}\right|^{2}\right) .
$$

On integrating Eq. (7) until the Fermi vector $k_{F}$ we find the charge densities in the partially occupied bands, namely, in the first and second conduction bands $\left(c_{A_{i}}\right.$ and $c_{B_{i}}$ depend also on the specific conduction band). When the interactions between the different atoms, which are expressed by the parameters $\gamma_{0}, \gamma_{1}, \gamma_{2}, \gamma_{3}, \gamma_{4}$, and $\gamma_{5}$, are taken into account the energy surface is not circular. In the presence of the external gate the electronic densities in fully occupied bands are not equal to each other and one needs to take into account also the density redistribution in the valence bands. Using Eqs. (3)-(5) and (7) we evaluate the energy gap $\Delta_{0}$ at the $K$ point, i.e., $k=0$ and the true gap, $\widetilde{\Delta}$, self-consistently following the approach in Refs. 12 and 13 for a fixed total density $n=n_{1}$ $+n_{2}+n_{3}$, which leads to a nonzero Fermi energy.

\section{B. Four-graphene layer system in an external electric field}

We consider a four-layer graphene system located near a positively charged gate, which induces a total excess density $n=n_{1}+n_{2}+n_{3}+n_{4}$, where $n_{i}$ is the excess density on the $i$ th layer as counted from the gate side. The corresponding changes in the potential energy between I and II, II and III, and III and IV layers are, respectively,

$$
\begin{gathered}
\Delta_{1,2}(n)=\alpha\left(n_{2}+n_{3}+n_{4}\right), \quad \Delta_{2,3}(n)=\alpha\left(n_{3}+n_{4}\right), \\
\Delta_{3,4}(n)=\alpha n_{4} .
\end{gathered}
$$

The Hamiltonian for the four $A B$ stacked graphene layers in the presence of an external electric field can be obtained by adding $\Delta_{1,2}(n), \Delta_{1,2}(n)+\Delta_{2,3}(n)$, and $\Delta_{1,2}(n)+\Delta_{2,3}(n)$ $+\Delta_{3,4}(n)$ to the II, III, and IV layer on-site elements of Eq. (1), respectively. The eight coefficients $c_{A_{i}}=c_{A_{i}}(\vec{k})$ and $c_{B_{i}}$ $=c_{B_{i}}(\vec{k})$ [for fixed values of the layer asymmetries defined by Eq. (8)] can be obtained by diagonalizing the Hamiltonian of the four-layer system. The electronic densities on the individual layers are given by Eq. (7). The gaps $\Delta_{0}$ and $\tilde{\Delta}$ are

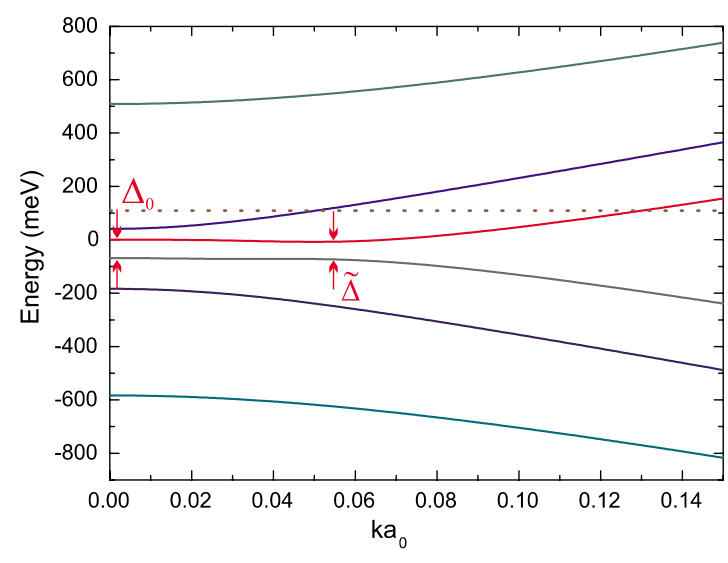

FIG. 2. (Color online) The band structure of three-layer graphene around the $K$ point for a total excess electron density $n$ $=10^{13} \mathrm{~cm}^{-2}$ in the case when we neglect all interactions except $\gamma_{0}$ and $\gamma_{1}$. Horizontal dotted line is the Fermi level.

evaluated self-consistently similarly as was done for the three-layer system.

\section{RESULTS}

First, we neglect all interactions except between the nearest-neighbor atoms in the same layer and between $A$-type atoms between adjacent layers (which are on top of each other), i.e., we put $\gamma_{2}=\gamma_{3}=\gamma_{4}=\gamma_{5}=\Delta=0$. In this approximation the energy bands are circular symmetric and shown in Fig. 2 for a gate voltage that induces a total electron density $n=10^{13} \mathrm{~cm}^{-2}$ for the three-layer graphene system. In our calculations we used the parameter $\gamma_{0}$ $=3.12 \mathrm{eV}$ which within each plane leads to an in-plane velocity $v=\sqrt{3} \gamma_{0} a / 2 \hbar \simeq 10^{6} \mathrm{~m} / \mathrm{s}$, and for the interlayer coupling strength, i.e., between $A_{i}$ and $A_{i+1}$ atoms, we take $\gamma_{1}$ $=0.37 \mathrm{eV}$ (see Ref. 10). For the dielectric constant we used the value $\kappa=1$ and for the interlayer distance $c_{0}=3.35 \AA$. Notice that for the considered electron density a part of the second conduction band lies below the Fermi level. In the bilayer case only the first conduction band is partially occupied $^{12}$ for such a density. The dependence of the Fermi energy $\varepsilon_{F}$ (dotted curve) as a function of the gate density $n$ is shown in Fig. 3 (when only $\gamma_{0}, \gamma_{1} \neq 0$, and all other $\gamma_{i}$ parameters are taken zero). The dependence of the energy gap at the $K$ point, $\Delta_{0}^{\prime}$ (dotted curve), is shown in Fig. 4 as a function of total excess density $n$ for the three-layer graphene (when only $\gamma_{0}, \gamma_{1} \neq 0$ ). The true gap $\widetilde{\Delta^{\prime}}$ (dashed curve) is also shown in Fig. 4. When comparing our results with those for bilayer graphene ${ }^{12}$ we notice that both $\Delta_{0}^{\prime}$ and $\widetilde{\Delta^{\prime}}$ gaps for the three-layer graphene case are much smaller as compared to the bilayer system for the same values of the total density. In the three-layer system $\widetilde{\Delta^{\prime}}=64.2 \mathrm{meV}$ and $\Delta_{0}^{\prime}$ $=68.4 \mathrm{meV}$ when $n=10^{13} \mathrm{~cm}^{-2}$, while this is $\widetilde{\Delta^{\prime}}$ $=141.5 \mathrm{meV}$ and $\Delta_{0}^{\prime}=152.7 \mathrm{meV}$ for bilayer graphene calculated using the values of $\gamma_{0}, \gamma_{1}$ chosen in our paper.

Now, we take into account all the interactions between the different atoms, which are expressed by the parameters $\gamma_{0}$, $\gamma_{1}, \gamma_{2}, \gamma_{3}, \gamma_{4}, \gamma_{5}$ and $\Delta$ (see Ref. 10). We use the values for 


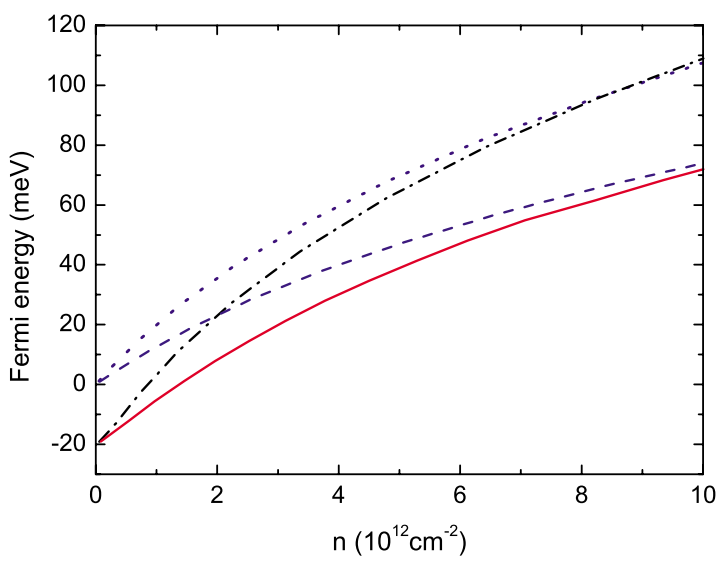

FIG. 3. (Color online) The dependence of the Fermi energy $\varepsilon_{F}$ on the total density $n$ for three-layer graphene in the case when we neglect all interactions except $\gamma_{0}$ and $\gamma_{1}$ (dotted curve) and include all interactions (dotted-dashed curve). The dependence for $\varepsilon_{F}$ for the four-layer graphene when we neglect all interactions except $\gamma_{0}$ and $\gamma_{1}$ (dashed curve) and for the full interaction case (solid curve).

graphite, as was done in Ref. 10. Although the exact values for these parameters may differ from graphite and for different number of layers, this choice will give a realistic idea of the effects due to the interaction with the other atoms. Figure 5 shows a three-dimensional (3D) plot and a contour plot of the first conductivity band for the three-layer graphene near the $K$ point ( $K$ point is chosen as the origin) which in this case has a triangular symmetry. For this case the Fermi energy dependence (dotted-dashed curve) as a function of the gate density $n$ is also shown in Fig. 3. The dependencies of $\widetilde{\Delta}$ (solid curve) and $\Delta_{0}$ (dotted-dashed curve) as a function of $n$ are shown in Fig. 4.

When the total excess density of electrons tends to zero the results obtained in the present work coincide with those found in Ref. 8. For example, for the three-layer graphene, for the case when all the SWMcC parameters are taken into

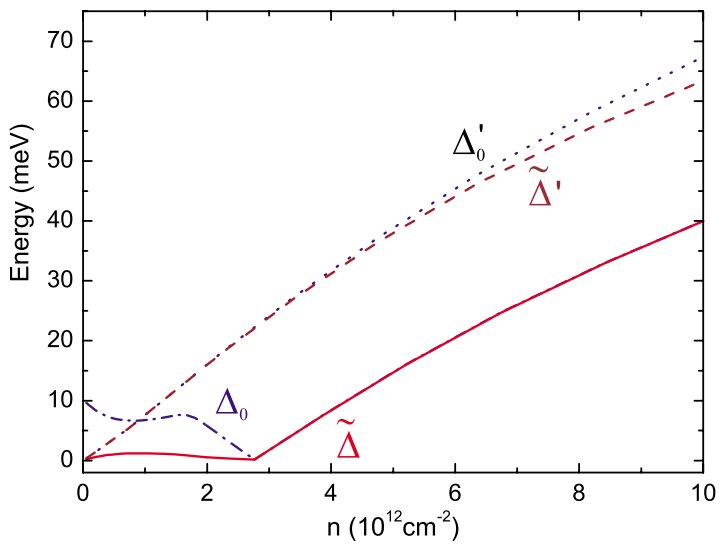

FIG. 4. (Color online) The dependence of the gap $\Delta_{0}$ at the $K$ point (dotted-dashed curve) and the true gap $\tilde{\Delta}$ (solid curve) for three-layer graphene when including the full interaction as a function of the total excess electron density $n$. For comparison we show also the corresponding results, $\Delta_{0}^{\prime}$ (dotted curve) and $\widetilde{\Delta^{\prime}}$ (dashed curve), when only $\gamma_{0}$ and $\gamma_{1} \neq 0$.

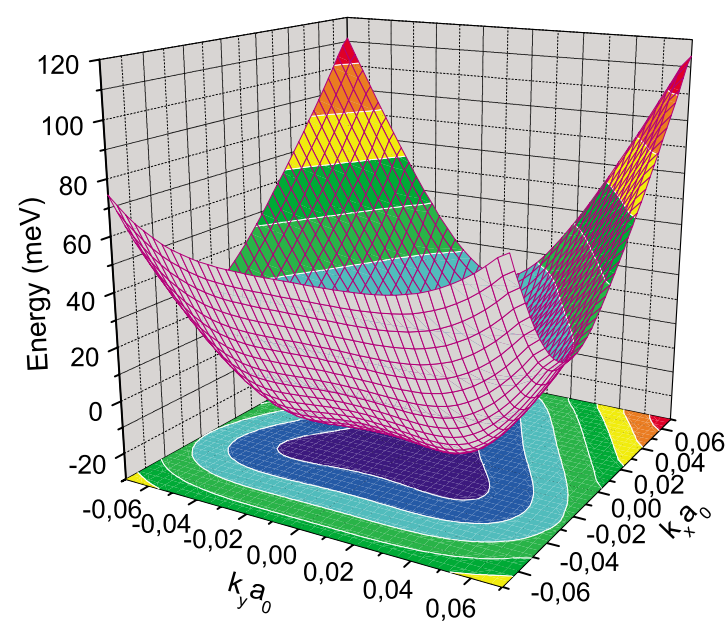

FIG. 5. (Color online) The first conductivity band and the contour plot for three-layer graphene with the full interaction included around the $K$ point for a total excess electron density $n=1.5$ $\times 10^{12} \mathrm{~cm}^{-2}$

account, the gap near the $K$ point equals $10 \mathrm{meV}$, which is in agreement with Ref. 8, and the other gaps equal 0 . The Fermi energy for the full interaction case is approximately $-20 \mathrm{meV}$ in the absence of external gates.

We have found that the energy gap at the $K$ point, $\Delta_{0}$, as well as the true gap, $\widetilde{\Delta}$, have a nonmonotonic behavior for the three-layer graphene with the full interaction between the atoms and that the curves coincide starting from intermediate densities. Figure 6 shows the typical band structures along $k_{x}$ for $k_{y}=0$, of three-layer graphene with the full interaction, for four different densities. The relatively small value of the true gap at low densities can be explained by the anticrossing of the third valence and first conduction bands in Fig. 6. The coincidence of the energy gap at the $K$ point with the true gap at intermediate and high densities can be explained by the shape of the lowest conduction and the highest valence band: the maximum of the highest valence band and the minimum of the lowest conduction band coincide at the $K$ point. The layer densities $n_{i}$ (solid curves) for the system with full interaction and for the case when only $\gamma_{0}, \gamma_{1} \neq 0$ (dashed curves) as a function of the total excess density $n$ are shown in Fig. 7.

It is interesting that the inclusion of a few extra small interaction parameters in the tight-binding (TB) Hamiltonian can lead to such big changes. The reason is the following and can already be understood from the results in the absence of a gate voltage. As you can see in Fig. 1 of Ref. 8, the inclusion of similar extra TB parameters that take into account extra interactions between atoms does not change the spectrum of bilayer graphene much. However, it changes the spectrum of a system with more than three layers in a more fundamental way. In the same figure in Ref. 8 it can be seen that the inclusion of the extra parameters leads to a decrease in the energy of the parabolic bands with respect to the linear bands. In other words, the ordering of the bands is changed. As a consequence, the energy gaps for the case with only two nonzero TB parameters and the case with all seven parameters are calculated between different bands: in the former 

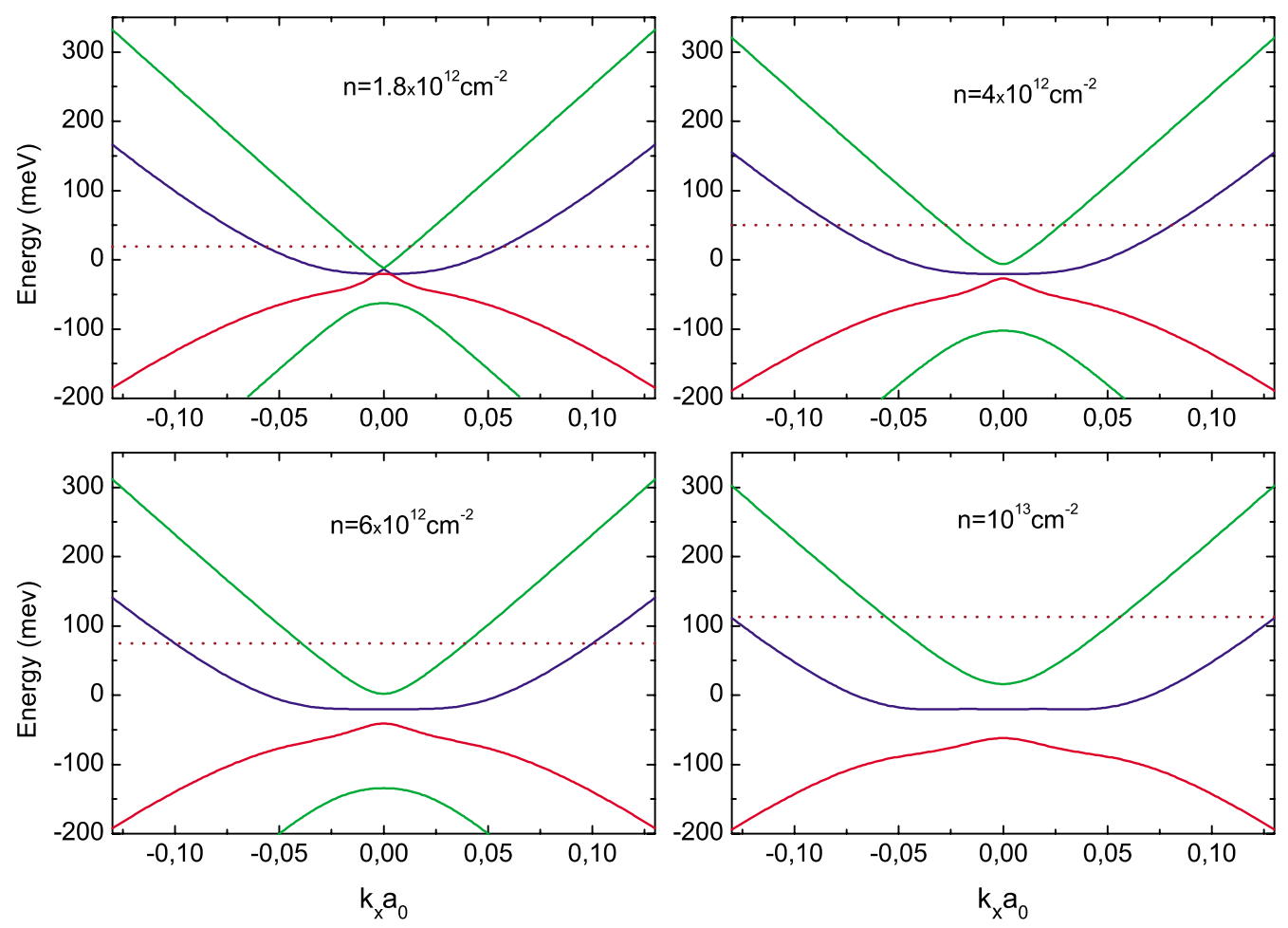

FIG. 6. (Color online) The band structure of the three-layer graphene with the full interaction as a function of $k_{x}$ (around the $K$ point for $\left.k_{y}=0\right)$ for different values of the total density. The horizontal dotted line corresponds to the Fermi energy.

case between the two parabolic bands (being the third and fourth levels at the $K$ point) and in the latter case between a linear (being the third level at the $K$ point) and a parabolic band (being the fourth level at the $K$ point). As many bands cross in multilayer graphene systems around the Fermi energy at the $K$ point, the inclusion of extra but small TB parameters may have a significant effect on the gaps.

We have also investigated the dependence of the $\widetilde{\Delta}$ and $\Delta_{0}$ for bilayer graphene with the full interaction. We have found that in the region of low densities, there is no considerable difference with the results in Ref. 12 obtained for the case when only $\gamma_{0}$ and $\gamma_{1} \neq 0$. The difference is substantial at the

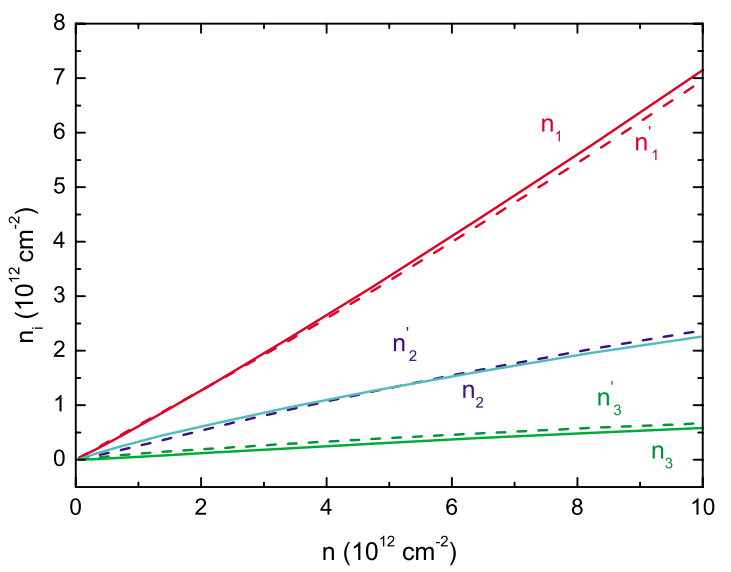

FIG. 7. (Color online) The layer densities $n_{i}$ (solid curves) for the three-layer system with the full interaction and $n_{i}^{\prime}$ (dashed curves) for the case when only $\gamma_{0}$ and $\gamma_{1}$ are different from zero, as a function of total excess density $n$. high densities; for $n=10^{13} \mathrm{~cm}^{-2}$ the true gap $\tilde{\Delta}$ $=124.2 \mathrm{meV}$ for the bilayer graphene with the full interaction while $\widetilde{\Delta^{\prime}}=141.5 \mathrm{meV}$ for the case when only $\gamma_{0}, \gamma_{1}$ $\neq 0$.

Finally, we consider a four-layer graphene system. The dependences of the Fermi energy $\varepsilon_{F}$ (solid curve) of the system with the full interaction and for the case when only $\gamma_{0}$, $\gamma_{1} \neq 0$ (dashed curve) as a function of the gate density $n$ are also shown in Fig. 3. The dependencies of $\widetilde{\Delta}$ (solid curve) and $\Delta_{0}$ (dashed curve) as a function of $n$ are shown in Fig. 8 . The same figure shows also the dependence of $\Delta_{0}^{\prime}$ (dotted-

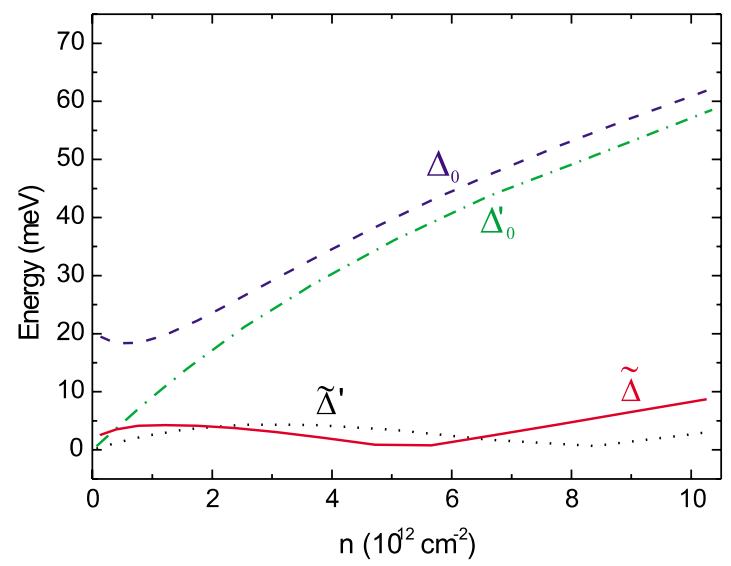

FIG. 8. (Color online) The dependences of $\widetilde{\Delta}$ (solid curve) and $\Delta_{0}$ (dashed curve) for the four-layer graphene system with the full interaction as a function of $n$ as well as for $\widetilde{\Delta^{\prime}}$ (dotted curve) and $\Delta_{0}^{\prime}$ (dotted-dashed curve) when only $\gamma_{0}$ and $\gamma_{1} \neq 0$. 

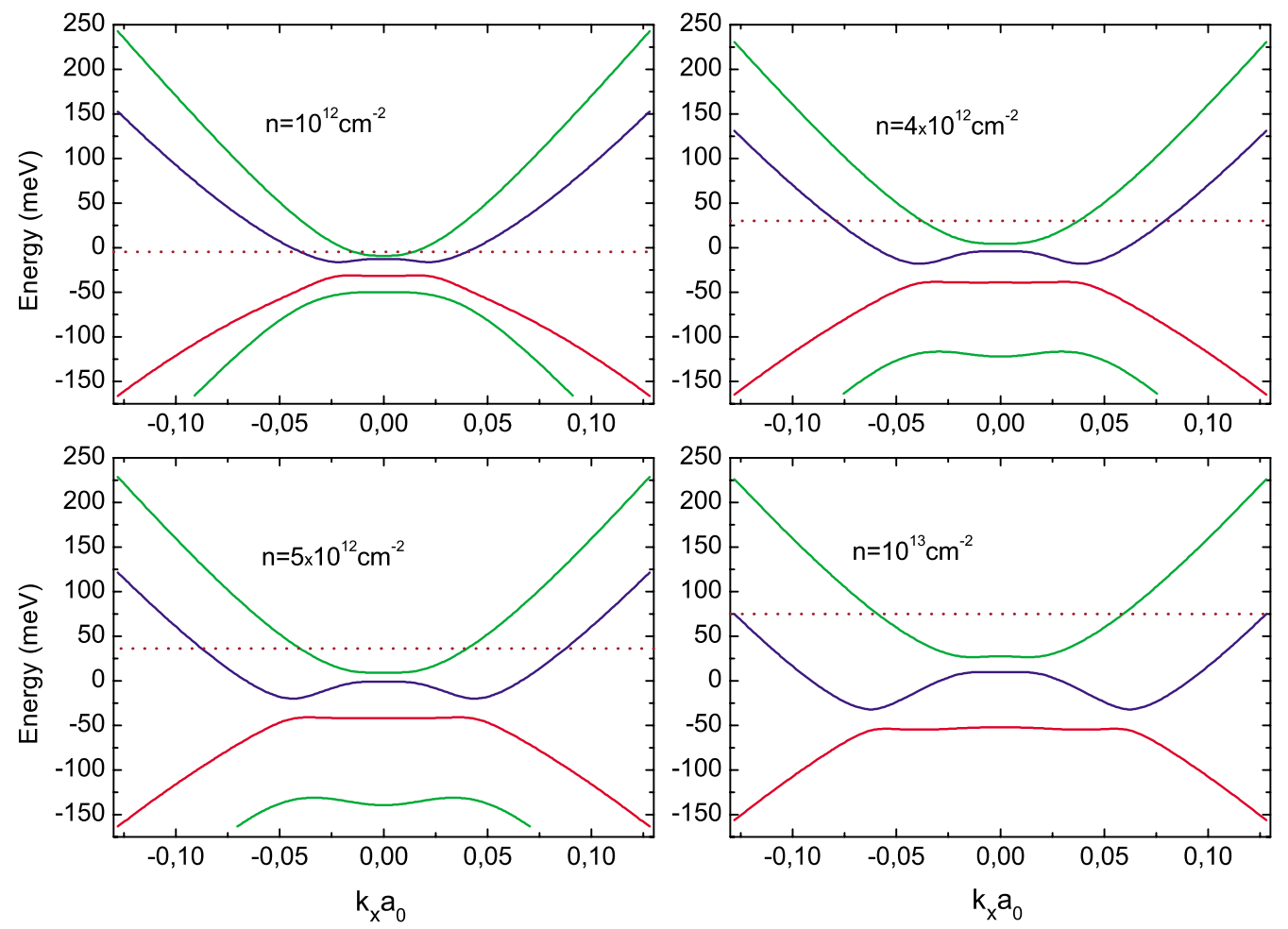

FIG. 9. (Color online) The band structure of the four-layer graphene with the full interaction as a function of $k_{x}$ (around the $K$ point for $\left.k_{y}=0\right)$ for different values of the total density (the horizontal dotted line corresponds to the Fermi energy).

dashed curve) and $\widetilde{\Delta^{\prime}}$ (dotted curve) for the case when only $\gamma_{0}, \gamma_{1} \neq 0$. Now, for the four-layer system we have found that the true gap has an oscillatory behavior as a function of $n$ for the situation when we include the full interaction as well as for the case when only $\gamma_{0}, \gamma_{1} \neq 0$. Figure 9 shows the typical band structure of the four-layer system with the full interaction along $k_{x}$ (for $k_{y}=0$ ) for different values of $n$; one can see that the true gap is much smaller than the gap near the $K$ point. However, the real value of the true gap, for fixed value of the total density, is determined by a gap with $k_{y} \neq 0$ and can be smaller than the values shown in Fig. 9. Figure 10

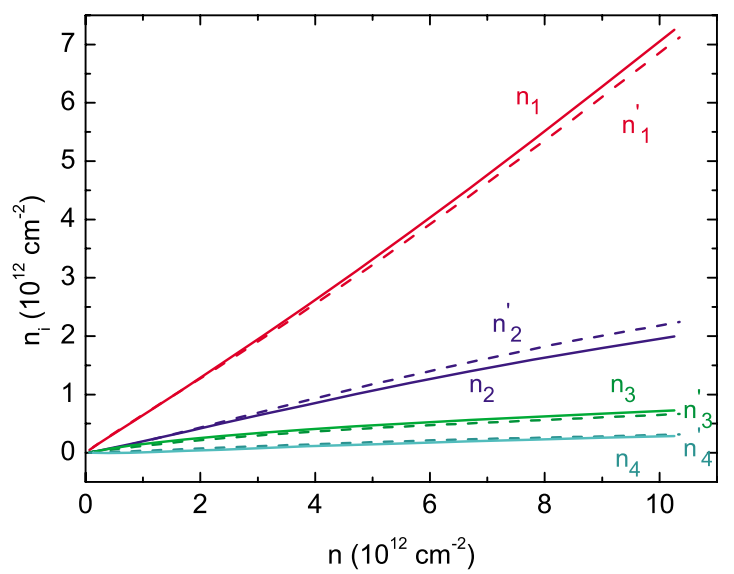

FIG. 10. (Color online) The layer densities $n_{i}$ (solid curves) for the four-layer graphene with the full interaction and $n_{i}^{\prime}$ (dashed curves) for the case when only $\gamma_{0}$ and $\gamma_{1} \neq 0$ as a function of total excess density $n$. shows the layer densities $n_{i}$ (solid curves) for the four-layer graphene with the full interaction and for the case when only $\gamma_{0}, \gamma_{1} \neq 0$ (dashed curves) as a function of total excess density $n$. The comparison of the layer densities $n_{i}$ for the fourlayer graphene (solid curves) and for three-layer graphene (dashed curves) as a function of total excess density $n$ (for the full interaction case) is shown in Fig. 11.

This behavior of the true gap is connected with the excess charge distribution and corresponding asymmetries between the graphene layers. Due to imperfect screening, the charge density on the layers farthest from the gate is considerably smaller in comparison with the excess density on the closest

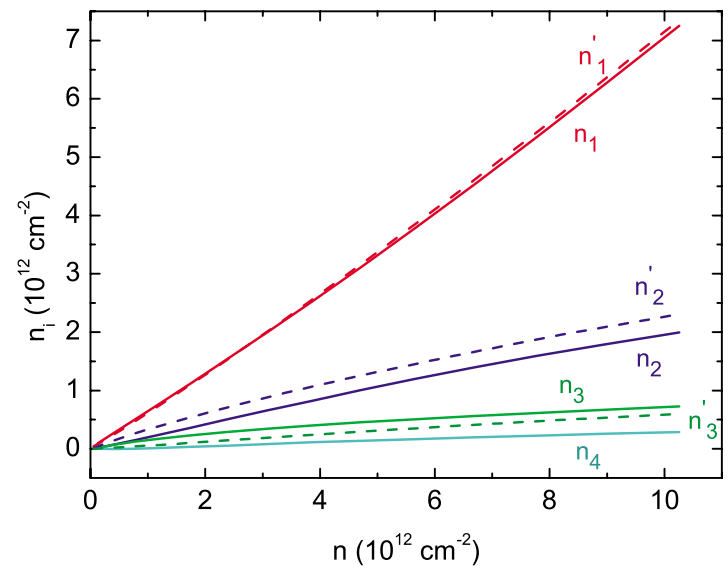

FIG. 11. (Color online) The layer densities $n_{i}$ for the four- (solid curves) and $n_{i}^{\prime}$ (dashed curves) for three-layer graphene systems respectively, as a function of total excess density $n$ (for the full interaction case). 
layer as it is shown in Figs. 7 and 10. With the increase in the number of layers, the asymmetry between the layers farthest away from the gate abruptly decreases, resulting in layers with almost no excess charge. As a result we have observed a tendency of a decrease in the true gap in the cases of intermediate and high total densities. Therefore it is expected that in the case when the number of layers $N_{l} \rightarrow \infty$ the gap will also tend to zero.

\section{CONCLUSIONS}

We considered the influence of a single gate placed above three- as well as four-layer graphene systems on the electronic structure near the $K$ point. We found that the gateinduced true gap for the three-layer system with full interaction is three times smaller as compared to the bilayer case for an external density $n=10^{13} \mathrm{~cm}^{-2}$. For the same large value of density the true gap for the four-layer system is about ten times smaller than in the bilayer case.

We have found that the energy gap at the $K$ point $\Delta_{0}$ as well as the true energy gap $\widetilde{\Delta}$ is a nonmonotonous function of the total density when including the full interaction between the atoms for the three-layer system. For the four-layer system we have found that only the true gaps oscillate as a function of the gate voltage both when including the full interaction and for the case when only $\gamma_{0}, \gamma_{1} \neq 0$.

We found the remarkable effect that for certain values of the gate voltage the gap can be closed. The re-entrant opening and closing of the energy gap were not seen in bilayer graphene and are typical for multilayer graphene. Note that those energy gaps are found in the band structure which is situated below the Fermi energy. Therefore, they will have a minor effect on electrical transport but will be detectable by optical measurements.

\section{ACKNOWLEDGMENTS}

This work was supported by the Flemish Science Foundation (FWO-Vl) and the "Belgian Science Policy," IAP program. Discussions with McCann are gratefully acknowledged.
*Permanent address: Yerevan State University, 1 A. Manoogian, 0025 Yerevan, Armenia; artak.avetisyan@ua.ac.be

†bart.partoens@ua.ac.be

*francois.peeters@ua.ac.be

${ }^{1}$ K. S. Novoselov, A. K. Geim, S. V. Morozov, D. Jiang, M. I. Katsnelson, I. V. Grigorieva, S. V. Dubonos, and A. A. Firsov, Nature (London) 438, 197 (2005).

${ }^{2}$ A. K. Geim and K. S. Novoselov, Nature Mater. 6, 183 (2007).

${ }^{3}$ P. Blake, P. D. Brimicombe, R. R. Nair, T. J. Booth, D. Jiang, F. Schedin, L. A. Ponomarenko, S. V. Morozov, H. F. Gleeson, E. W. Hill, A. K. Geim, and K. S. Novoselov, Nano Lett. 8, 1704 (2008).

${ }^{4}$ S. V. Morozov, K. S. Novoselov, F. Schedin, D. Jiang, A. A. Firsov, A. K. Geim, Phys. Rev. B 72, 201401(R) (2005).

${ }^{5}$ Y. Zhang, Y.-W. Tan, H. L. Stormer, and P. Kim, Nature (London) 438, 201 (2005).
${ }^{6}$ C. Berger, Z. Song, X. Li, X. Wu, N. Brown, C. Naud, D. Mayou, T. Li, J. Hass, A. N. Marchenkov, E. H. Conrad, Ph. N. First, and W. A. de Heer, Science 312, 1191 (2006).

${ }^{7}$ I. A. Luk'yanchuk and Y. Kopelevich, Phys. Rev. Lett. 93, 166402 (2004); 97, 256801 (2006).

${ }^{8}$ B. Partoens and F. M. Peeters, Phys. Rev. B 75, 193402 (2007).

${ }^{9}$ S. Latil and L. Henrard, Phys. Rev. Lett. 97, 036803 (2006).

${ }^{10}$ B. Partoens and F. M. Peeters, Phys. Rev. B 74, 075404 (2006).

${ }^{11}$ T. Ohta, A. Bostwick, T. Seyller, K. Horn, and E. Rotenberg, Science 313, 951 (2006).

${ }^{12}$ E. McCann, Phys. Rev. B 74, 161403(R) (2006).

${ }^{13}$ E. V. Castro, K. S. Novoselov, S. V. Morozov, N. M. R. Peres, J. M. B. Lopes dos Santos, J. Nilsson, F. Guinea, A. K. Geim, and A. H. Castro Neto, Phys. Rev. Lett. 99, 216802 (2007).

${ }^{14}$ J. Güttinger, C. Stampfer, F. Molitor, D. Graf, T. Ihn, and K. Ensslin, New J. Phys. 10, 125029 (2008). 